\title{
FOXP3/CD8 ratio and CXCR4-positive regulatory T cells as indicators of tumor immunity in the conjunctival squamous cell carcinoma microenvironment
}

\author{
Mizuki Tagami ( $\nabla$ tagami.mizuki@med.osaka-cu.ac.jp ) \\ Osaka City University \\ Anna Kakehashi \\ Osaka City University \\ Atsuko Katsuyama-Yoshikawa \\ Kobe Kaisei Hospital
}

Atsushi Sakai

Osaka City University

Norihiko Misawa

Osaka City University

Hideki Wanibuchi

Osaka City University

Atsushi Azumi

Kobe Kaisei Hospital

Shigeru Honda

Osaka City University

\section{Research Article}

Keywords: Conjunctival squamous cell carcinoma, regulatory T cell, FOXP3, tumor-infiltrating lymphocyte,CXCR4, tumor microenvironment

Posted Date: March 5th, 2021

DOI: https://doi.org/10.21203/rs.3.rs-265946/v1

License: (9) (1) This work is licensed under a Creative Commons Attribution 4.0 International License. Read Full License 


\section{Abstract}

Conjunctival squamous cell carcinoma (SCC) is the most common ocular surface neoplasia. The purpose of this retrospective study was to examine the role of regulatory $T$ cells (Tregs) activity in tumor immunity and investigate the tumor microenvironment as a new treatment focus in conjunctival SCC.

A cancer progression gene array analysis and Immunohistochemical analysis of FOXP3 as Treg marker, CD8 as Tumor-infiltrating lymphocyte marker, and CXCR4 expression on activate Treg was also examined in a series of 31 conjunctival SCC cases. To investigate the localization of FOXP3-positive Tregs in detail, the tumor surface, tumor central, and around vessels staining patterns of FOXP3, CD8, and CXCR4 were examined separately.

A significant difference in FOXP3, CD8, and CXCR4 staining in tumor-infiltrating lymphocytes and FOXP3/CD8 ratio were in the carcinoma in situ group than advanced stage SCC group(each $P<0.01)$. In addition, FOXP3/CD8 ratio was correlation with progression-free survival. Double immunostaining of CXCR4/FOXP3 correlate with American Joint Committee on Cancer T-stage, independent of age or Ki67 index $(P \otimes 0.01)$. Our results show that FOXP3, the FOXP3/CD8 ratio, and the CXCR4 axis are important pathologic and prognostic factors of ocular surface neoplasia, including SCC. These tumor microenvironment of conjunctival SCC may be considered in the future development of treatment options.

\section{Introduction}

Ocular surface squamous neoplasia (OSSN) includes several diseases, such as conjunctival premalignant dysplasia, carcinoma in situ, and invasive conjunctival squamous cell carcinoma (SCC) ${ }^{1}$. The annual incidence of OSSN in the United Kingdom is 0.53 cases/million population/year (conjunctival intraepithelial neoplasia: 0.43 cases/million population/year; SCC: 0.08 cases/million population/year) ${ }^{2}$, ${ }^{3}$. In the United States, the incidence of SCC is 5 -fold higher among males and whites ${ }^{4}$. However, no large-scale epidemiologic studies have been performed in populations in the Far East. In addition, recent studies have reported associations between findings such as loss of retinoblastoma protein/P16/cyclin D1, strong p53 immunostaining, and ADAM3A overexpression and the molecular pathology of SCC ${ }^{5}{ }_{-}^{6}$. In recent years, research on factors related to tumor immunity, including programmed cell death 1 (PD-1), programmed cell death ligand 1 (PDL-1), and cytotoxic T lymphocyte-associated protein 4 (CTLA-4) has advanced worldwide to encompass aspects ranging from basic research to clinical indications in headneck SCC ${ }^{7},{ }^{8-10}$. Recent ophthalmologic-related research demonstrated that PDL-1 is expressed in almost half of conjunctival SCC cases and indicated the potential application of immune checkpoint blockade as a treatment strategy for conjunctival SCC. Other research indicates that a significant number of sebaceous carcinoma cases involve expression of PDL- 1 at therapeutic levels with tumor-infiltrating $T$ cells 611 
T regulatory cells (Tregs) play a major role in tumor immunity in the tumor microenvironment ${ }^{12-15}$. In particular, CD4(+) Tregs expressing the transcription factor forkhead box P3 (FOXP3) are abundant in tumor tissues, where they appear to hinder the induction of effective antitumor immunity ${ }^{12}$. Tregs function in maintaining immunologic self-tolerance by actively suppressing self-reactive lymphocytes, and the gene encoding FOXP3 is a key regulator of Treg development ${ }^{16}$. Freeman et al. reported that a high CD $4+T$ cell to $C D 8+T$ cell ratio may be an immunologic diagnostic indicator of late-stage dermal SCC development in immune-competent patients ${ }^{17}$. Considerable research attention has focused on the relationship between the role of local tumor-infiltrating lymphocytes (TILs [CD $4+$ and/or CD8 + T cells]) associated with FOXP3 + Tregs in the tumor microenvironment and the prognosis of SCC patients ${ }^{9}$. Elucidating details regarding the interactions between Tregs in the tumor microenvironment and other molecules is another important area of research. The present study investigated the expression of FOXP3 in tumor tissues from SCC patients in order to assess the molecular association between Tregs and prognosis in East Asian patients with conjunctival SCC.

\section{Materials And Methods}

\section{Selection of Cases and Collation of Clinicopathologic Data}

This study was approved by the Institutional Review Boards of Osaka City University and Kobe Kaisei Hospital and adhered to the tenets of the 1964 Declaration of Helsinki.

Written informed consent was obtained from all patients before enrollment.

We identified 29 patients treated by ophthalmologists (AA, MT) between November 2007 and July 2018 from whom we were able to procure tissue blocks with residual tumors. Demographic information (age at initial diagnosis and at presentation to our institution; sex) and primary tumor features (disease status at presentation [primary or recurrent] and in situ versus invasive disease) were obtained from each patient enrolled. The American Joint Committee on Cancer (AJCC) stage, local recurrence (anatomic site and date), metastases (regional or distant, and date), vital status at last follow-up, cause of death if deceased, type of surgery, and adjuvant therapy were also recorded.

\section{Immunohistochemistry}

Immunohistochemical analyses of FOXP3 and CD8 expression were performed on 5- $\mu$ m-thick tissue sections using the following antibodies: anti-human FOXP3 mouse monoclonal (clone: 236A/E7; \#ab20034; Abcam, Cambridge, UK), anti-human CD8 mouse monoclonal (clone: 4B11; NCL-L-cd8-4B11; Leica, Newcastle, UK), anti-human CXCR4 rabbit polyclonal (NB100-74396; Novus, Continental, CO), Elite ABC Rabbit kit and Elite ABC Mouse kit (PK-6101, PK-6102; Vector Laboratories, Burlingame, $C A$ ), and ABC-AP Mouse IgG kit (AK-5002; Vector Laboratories). Tissue sections were incubated in ImmPACT DAB (Vector Laboratories) and Alkaline Phosphatase Substrate kit III <VECTOR Blue> (Vector Laboratories) until the desired staining intensity developed. The sections were then counterstained with hematoxylin and mounted. Positive and negative staining controls for all antibodies were carried out in parallel using tonsillar tissue. Stained sections were viewed under an Olympus BX53+DP74 microscope. 


\section{Image Analysis}

Tissues immunostained for FOXP3, CD8, and CXCR4 were evaluated in a blinded manner by two specialists (MT and AK). The first field for evaluation in each tumor lesion was selected randomly, and subsequently, 10 fields were examined systematically at 400x magnification using a mesh.

FOXP3 and CD8 expression was analyzed visually as the presence or absence and intensity of cell staining, and samples were divided semi-quantitatively into groups based on a score of 0 to $3(0$, none:0:1 field; 1 , weak: $1-5>1$ field; 2 , strong: 5-10> 1 field; 3 , very strong $10>1$ field).

In order to investigate localization in greater detail, the FOXP3 and CD8 staining patterns in tissues were examined separately for the tumor surface (SF), center of the tumor (TC), and around vessels (AV). For FOXP3/CXCR4 double staining, the number of Tregs co-expressing FOXP3/CXCR4 was determined from four $(\times 40)$ high-power fields of lymphoid rich infiltrate within the tumor, according to previously reported methods $\left[{ }^{18}\right]$. Samples were semi-quantitatively divided into groups based on a FOXP3/CXCR4 double staining score in the range 0 to 3 ( 0 , none:0:1 field; 1 , weak: 1-2> 1 field; 2 : strong: $3>1$ field) according to the presence or absence and intensity of staining.

\section{Gene Expression in Tumors}

The expression of major cancer-associated genes in tumor tissues was compared between patients with carcinoma in situ and invasive SCC using NanoString analysis.

Genes examined included EGFR, HIF1A, ICAM1, IL-6, CXCR-

4, MAPK1, MMP2, MMP9, NFKB1, NOTCH1, ROCK1, ROCK2, TGFB1, TNF, VEGFA, and WNT5A. Archival formalin-fixed paraffin-embedded tumor tissues were retrieved and manually macrodissected. Total mRNA was isolated using a Qiagen miRNeasy kit (Qiagen, Valencia, CA, USA) according to the manufacturer's instructions. Isolated RNA was quantified using a NanoDrop system (Thermo Scientific, Wilmington, DE, USA) and regarded as adequate if a sample contained a minimum of $400 \mathrm{ng}$. The samples were subsequently analyzed using an nCounter PanCancer Progression Panel (NanoString, Seattle, WA, USA) according to the manufacturer's instructions. NanoString data were processed using the R statistical programming environment (v3.4.2). Considering counts obtained for positive control probe sets, raw NanoString counts for each gene were subjected to technical factorial normalization, which was carried out by subtracting the mean count plus two standard deviations from the CodeSet inherent negative controls. Subsequently, biological normalization using the included mRNA reference genes was performed. Additionally, all counts with $P>0.05$ by one-sided $t$-test versus negative controls plus two standard deviations were interpreted as not expressed over basal noise.

\section{Statistical Analyses}

Clinical and histopathologic characteristics were summarized using descriptive statistics. Correlations between immunohistochemical, demographic, and clinicopathologic factor data were assessed using the 
Wilcoxon rank sum and Fisher's exact tests. Analysis of covariance was used to analyze the relationship between T-stage of tumors and FOXP3/CXCR4-positive Tregs. With respect to regression analyses, an explanatory variable that roughly divided the number of cases by 15 was considered appropriate ${ }^{19}$. Progression-free survival (PFS) was defined as the time from surgery to disease recurrence or death from any cause. Cox regression modeling was used to evaluate correlations between clinicopathologic and immunohistochemical features and survival outcomes. Statistical analyses were performed using SPSS Statistics software, version 22 (IBM Japan, Tokyo, Japan). $P<0.05$ was considered indicative of statistical significance.

\section{Results}

Clinicopathologic findings of our cohort are summarized in Table 1. All 31 patients (100\%) were East Asian and included 17 men and 14 women, with a mean age at presentation of 77.9 years. Sixteen patients (51\%) had invasive SCC, and $15(49 \%)$ had an in situ tumor. Primary orbital exenteration was necessary for local disease control in three patients (9\%), and two patients (6\%) underwent additional orbital exenteration. Nine patients $(29 \%)$ underwent adjuvant therapy, most commonly additional local surgery. Topical chemotherapy and radiation therapy were performed in one patient in the adjuvant therapy group. One patient in the adjuvant therapy group died with disease 11 months after diagnosis of regional and lung metastases; the another patientwas alive without disease at 50 months after diagnosis of regional metastases. Three patients ( $9 \%$ ) died, two of which was due to conjunctival SCC (described above). Nine patients (31\%) experienced local recurrence after curative surgery (Table 1 ).

Twenty-two tumors in our cohort (70\%) were FOXP3 positive (Fig. 1a, b). wenty-two tumors were FOXP3 positive $(70 \%)$ in our cohort(figure.1A,B). There were Three of 15 tumors $(20 \%)$ showed FOXP3 SF staining in Tis(score: $0.20 \pm 0.32$ ), six of 15 tumors (40\%) showed FOXP3 TC staining in Tis(score:0.46 \pm 0.56), and six of 15 tumors (40\%) showed FOXP3 AV staining in Tis(score:1.0 \pm 0.93 ). There were eleven of 16 tumors $(68 \%)$ showed FOXP3 SF staining in Tadv(score:0.86 $\pm 0.92, P<0.01$ ), thirteen of 16 tumors (86\%) showed FOXP3 TC staining in Tadv(score:2.13 $\pm 2.07, \mathrm{P}<0.01)$, and fourteen of 16 tumors $(77 \%)$ showed FOXP3 AV staining in Tadv(score:2.60 $\pm 2.57, \mathrm{P}<0.01)$ (table.2).

In CD8 staining, All 19 tumors were CD8 positive (61\%) in each local staining pattern in our cohort(figure.1C,D). There were five of 15 tumors (33\%) showed CD8 SF staining in Tis(score:0.60 \pm 0.72 ), nine of 15 tumors (60\%) showed CD8 TC staining in Tis(score:0.86 \pm 0.69$)$, and nine of 15 tumors (60\%) showed CD8 AV staining in Tis(score:2.0 \pm 0.40 ). There were four of 16 tumors $(25 \%)$ showed CD8 SF staining $n$ Tadv(score: $0.20 \pm 0.21, P=0.12$ ), six of 16 tumors (40\%) showed CD8 TC staining in $\operatorname{Tadv}$ (score:0.60 $\pm 0.64, P \otimes 0.35$ ), and eight of 16 tumors (53\%) showed CD8 AV staining in Tadv(score:1.26 $\pm 1.28, P \llbracket 0.01$ ) (table.2). Furthermore, FOXP3/CD8 ratio were significant difference only in $\mathrm{AV}$ group $(\mathrm{P}<0.01)($ Table.3)

Expression of EGFR, HIF1A, ICAM1, IL-6, CXCR4, MAPK1, MMP2, MMP9, NFKB1, NOTCH1, ROCK1, ROCK2, TGFB1, TNF, VEGFA, and WNT5A was compared between the Tis and Tadv groups according to AJCC T 
grading ( $\mathrm{n}=4$ in each group) (Table 4) and Fig. 2). Expression of mRNA for CXCR4, HIF-1a, IL-6, NFKB1, and ROCK1 differed significantly $(P<0.05)$ between the Tis and Tadv groups.

Double CXCR4/FOXP3 immunostaining was performed to evaluate CXCR4-positive Treg infiltration in whole samples. A higher proportion of Tadv tumors expressed CXCR (score: 1.43) compared with Tis tumors (score: 0.26$)(P<0.01)$ (Fig. 3). Analysis of covariance indicated that double CXCR4/FOXP3 immunostaining was also correlated with T-stage, independent of age and Ki67 index $(P<0.01)$.

The Cox regression model was used to examine the relationship between long-term prognosis (including orbital exenteration and PFS) and clinicopathologic status, FOXP3 and CD8 staining pattern, and the FOXP3/CD8 ratio. Univariate Cox regression analyses revealed significant correlations between the FOXP3/CD8 ratio and PFS (hazard ratio [HR]: 2.68; $P=0.049$ ) (Table 5). Local recurrence, distant metastasis rate, and overall survival rate were not significantly correlated. In addition, the FOXP3 and CD8 staining pattern and FOXP3/CD8 ratio were not significantly correlated with final orbital exenteration (data not shown).

\section{Discussion}

To the best of our knowledge, this is one of the first studies to investigate immunity in the tumor microenvironment in conjunctival SCC and evaluate the prognostic significance of TILs expressing FOXP3 and CXCR4 and Tregs comparing Tis with advanced SCC (Tadv).

In this study, we found a clear difference in TIL expression of FOXP3 between the Tis and Tadv groups. In addition, the FOXP3/CD8 ratio was determined to confirm the TIL balance in the tumor microenvironment. There was a significant difference in the AV FOXP3/CD8 ratio close to tumors between the Tis and Tadv groups. In addition, increased expression of CXCR4 in the Tadv group was confirmed by both immunohistochemical and mRNA expression analyses.

Recent research has shown that immune dysregulation, polyendocrinopathy, enteropathy, X-linked syndrome is caused by mutations in FOXP3, indicating this is the master gene of Tregs ${ }^{16},{ }^{20}{ }^{21}$. The main role of these in the microenvironment of tumor is Treg cells, many studies have been investigated FOXP3 expression and CD8 or other lymphocyte marker ratio in TIL as Treg cell marker ${ }^{12-15}$. In ophthalmologic research, some studies have reported that Tregs play a role in protecting tissues from autoimmune diseases, such as dry eye or graft versus host disease after bone marrow transplantation, as well as ocular malignant neoplasms ${ }^{22,23}$.

However, some studies in the field of systemic oncology have reported that Tregs block TILs in the tumor microenvironment, thus promoting tumor progression. Therefore, local suppression of Tregs in the tumor is considered a new axis of targeted treatment, and a number of studies examining this possibility are underway ${ }^{12-15}$. 
Our study is the first to examine the expression of FOXP3 and the FOXP3/CD8 ratio in conjunction with tumor progression and prognosis in patients with conjunctival SCC. This is a new finding regarding tumor escape from immunity, which was also shown in our case of conjunctival SCC. In addition, double immunostaining of CXCR4 and FOXP3 in conjunctival SCC indicated that these factors may play cooperative molecular roles. When a tumor progresses, the ratio of FOXP3 to CD8 expression shifts toward FOXP3. This is consistent with previous studies of SCC tumors in other areas and suggests that the change in FOXP3/CD8 ratio affects tumor growth by enabling tumor cells to evade attack by TIL in the tumor microenvironment ${ }^{7-9}$.

In order to investigate the mechanism by which tumor cells induce Tregs, we comprehensively examined the expression of genes thought to play a major role in tumor growth. We found that inflammation- and ischemia-related factors such as IL- 6 and HIF-1 a were overexpressed. Previous studies have reported that these factors induce Tregs to proliferate and aggregate into tumors ${ }^{24}$. Furthermore, vascular endothelial growth factor (VEGF)-associated ischemia has been shown to inhibit dendritic cell maturation via the NFKB pathway, which would diminish the presentation of cancer cell antigens and promote the growth of cancer cells in the ocular microenvironment ${ }^{25}$. Indeed, in our study, expression of NF-KB was significantly lower in the Tdav group than the Tis group, although this was evaluated only at the mRNA level.

Among the genes examined, we were very interested in the expression of $C X C R 4$, as CXCR4 is reportedly associated with tumor growth, and some studies indicated that CXCR4 affects the proliferation and activity of Tregs in the tumor microenvironment ${ }^{26-29}$.

In addition, a relationship between CXCR4 and FOXP3-positive Tregs has been reported ${ }^{18}$. The results of our study also suggest that IL- 6 and HIF- $1 a$ are associated with CXCR4 and that CXCR4 expression may be associated with an increase in FOXP3-positive Tregs. This means that inflammation is the anti-tumor immune effect of CXCR4-axis Tregs due to ischemia, and to our knowledge, ours is the first report of this association in the area of conjunctival SCC (Fig. 4).

These findings have important implications in SCC, which is strongly resistant to targeted therapies but could potentially respond to immunotherapies that target Tregs. Furthermore, it is possible that Treg recruitment by CXCR4 in these cancers could be modulated by treatment directed against hypoxia pathway factors, including HIF-1a(Figure.4). Thus, the development of treatment strategies based on reagents directed against Tregs, antibodies that block CXCR4, or inhibitors of HIF-1a and VEGF represents a potentially fruitful area of research.

This study has important limitations. First, regarding FOXP3 and CXCR4 expression in OSSN, changes associated with benign disease and age-related changes in normal tissues may not have been sufficiently investigated. Further studies, including multi-institutional studies and an increase in the number of cases, will be needed in the future. In addition, the size of our study cohort was small $(\mathrm{N}=31)$, and the length of follow-up (less than 1 year in some patients) may not have been sufficient for long-term outcome analyses. Therefore, additional studies will be needed to corroborate our findings. 
In conclusion, the results of this study indicate that FOXP3 and CXCR4, which are indicators of Treg activity, are potential molecular targets and prognostic factors in the treatment of OSSN, including SCC. Our findings also suggest that the tumor microenvironment of conjunctival SCC must be considered in the future development of treatment options.

\section{Declarations}

\section{Funding}

None of the authors have any proprietary or financial interests to declare.

Competing interests

The authors declare that they have no competing interests.

Ethics declarations

Ethics approval and consent to participate

All procedures performed in studies involving human participants were conducted in accordance with the ethical standards of the Institutional and/or National Research Committee and with the 1964 Declaration of Helsinki and its later amendments or comparable ethical standards.

Approval for this study was obtained prior to the start of the study from the institutional review board at Osaka City University, Japan (IRB-4236). Written informed consent for the storage of patient information in the hospital database and use in research was provided by all patients enrolled in the study.

Consent for publication

Not Applicable.

Contributions

MT wrote the main text of the manuscript and prepared the figures. Datasets were prepared by NM and AS. AK, HW, and $\mathrm{SH}$ reviewed the manuscript and checked the statistical analysis. All authors have read and approved the manuscript in its final form.

Data Sharing

All data relevant to the study are included in the article or uploaded as online supplementary material.

\section{Acknowledgements}

We gratefully acknowledge the technical assistance of the Research Support Platform, Osaka City University Graduate School of Medicine, and the Clinical Laboratory Department of Kobe Kaisei Hospital. 


\section{References}

1 Lee, G. A. \& Hirst, L. W. Ocular surface squamous neoplasia. Surv. Ophthalmol. 39, 429-450, doi:10.1016/s0039-6257(05)80054-2 (1995).

$2 \quad$ Kiire, C. A. et al. A prospective study of the incidence, associations and outcomes of ocular surface squamous neoplasia in the United Kingdom. Eye (Lond.) 33, 283-294, doi:10.1038/s41433-0180217-x (2019).

3 McClellan, A. J. et al. Epidemiology of Ocular Surface Squamous Neoplasia in a Veterans Affairs Population. Cornea 32, 1354-1358, doi:10.1097/IC0.0b013e31829e3c80 (2013).

4 Sun, E. C., Fears, T. R. \& Goedert, J. J. Epidemiology of squamous cell conjunctival cancer. Cancer Epidemiol. Biomarkers Prev. 6, 73-77 (1997).

5 Chauhan, S. et al. Loss of pRB in Conjunctival Squamous Cell Carcinoma: A Predictor of Poor Prognosis. Appl. Immunohistochem. Mol. Morphol. 26, e70-e76, doi:10.1097/pai.0000000000000592 (2018).

6 Vizcaino, M. A. et al. ADAM3A copy number gains occur in a subset of conjunctival squamous cell carcinoma and its high grade precursors. Hum. Pathol. 94, 92-97, doi:10.1016/j.humpath.2019.08.020 (2019).

7 Iwai, Y. et al. Involvement of PD-L1 on tumor cells in the escape from host immune system and tumor immunotherapy by PD-L1 blockade. Proceedings of the National Academy of Sciences 99, 1229312297, doi:10.1073/pnas.192461099 (2002).

8 Harrington, K. J. et al. Nivolumab versus standard, single-agent therapy of investigator's choice in recurrent or metastatic squamous cell carcinoma of the head and neck (CheckMate 141): healthrelated quality-of-life results from a randomised, phase 3 trial. Lancet Oncol. 18, 1104-1115, doi:10.1016/s1470-2045(17)30421-7 (2017).

9 Ferris, R. L. et al. Nivolumab for Recurrent Squamous-Cell Carcinoma of the Head and Neck. N. Engl. J. Med. 375, 1856-1867, doi:10.1056/NEJMoa1602252 (2016).

10 Leach, D. R., Krummel, M. F. \& Allison, J. P. Enhancement of Antitumor Immunity by CTLA-4 Blockade. Science 271, 1734-1736, doi:10.1126/science.271.5256.1734 (1996).

11 Saliba, M. et al. PD-L1 expression in sebaceous carcinomas. Cancer Immunol. Immunother., doi:10.1007/s00262-020-02821-3 (2021). 
12 Sugiyama, D. et al. Anti-CCR4 mAb selectively depletes effector-type FoxP3+CD4+ regulatory T cells, evoking antitumor immune responses in humans. Proc. Natl. Acad. Sci. U. S. A. 110, 17945-17950, doi:10.1073/pnas.1316796110 (2013).

13 Spranger, S., Bao, R. \& Gajewski, T. F. Melanoma-intrinsic $\beta$-catenin signalling prevents antitumour immunity. Nature 523, 231-235, doi:10.1038/nature14404 (2015).

14 Shimizu, J., Yamazaki, S. \& Sakaguchi, S. Induction of Tumor Immunity by Removing CD25<sup $>+</$ sup $>$ CD $4<$ sup $>+</$ sup $>$ T Cells: A Common Basis Between Tumor Immunity and Autoimmunity. The Journal of Immunology 163, 5211-5218 (1999).

15 Nishikawa, H. \& Sakaguchi, S. Regulatory T cells in tumor immunity. Int. J. Cancer 127, 759-767, doi:10.1002/ijc.25429 (2010).

16 Hori, S., Nomura, T. \& Sakaguchi, S. Control of Regulatory T Cell Development by the Transcription Factor <em>Foxp3</em>. Science 299, 1057-1061, doi:10.1126/science.1079490 (2003).

17 Freeman, A. et al. Comparative immune phenotypic analysis of cutaneous Squamous Cell Carcinoma and Intraepidermal Carcinoma in immune-competent individuals: proportional representation of CD8+ T-cells but not FoxP3+ Regulatory T-cells is associated with disease stage. PLoS One 9, e110928, doi:10.1371/journal.pone.0110928 (2014).

18 Yan, M. et al. Recruitment of regulatory T cells is correlated with hypoxia-induced CXCR4 expression, and is associated with poor prognosis in basal-like breast cancers. Breast Cancer Res. 13, R47, doi:10.1186/bcr2869 (2011).

19 Peduzzi, P., Concato, J., Kemper, E., Holford, T. R. \& Feinstein, A. R. A simulation study of the number of events per variable in logistic regression analysis. J. Clin. Epidemiol. 49, 1373-1379, doi:10.1016/s0895-4356(96)00236-3 (1996).

20 Bennett, C. L. et al. The immune dysregulation, polyendocrinopathy, enteropathy, X-linked syndrome (IPEX) is caused by mutations of FOXP3. Nat. Genet. 27, 20-21, doi:10.1038/83713 (2001).

21 Fontenot, J. D., Gavin, M. A. \& Rudensky, A. Y. Foxp3 programs the development and function of CD4+CD25+ regulatory T cells. Nat. Immunol. 4, 330-336, doi:10.1038/ni904 (2003).

22 Chauhan, S. K. et al. Autoimmunity in Dry Eye Is Due to Resistance of Th17 to Treg Suppression. The Journal of Immunology 182, 1247-1252, doi:10.4049/jimmunol.182.3.1247 (2009).

23 Socié, G. \& Ritz, J. Current issues in chronic graft-versus-host disease. Blood 124, 374-384, doi:10.1182/blood-2014-01-514752 (2014).

24 Gabrilovich, D. I. \& Nagaraj, S. Myeloid-derived suppressor cells as regulators of the immune system. Nat. Rev. Immunol. 9, 162-174, doi:10.1038/nri2506 (2009). 
25 Gabrilovich, D. I. et al. Production of vascular endothelial growth factor by human tumors inhibits the functional maturation of dendritic cells. Nat. Med. 2, 1096-1103, doi:10.1038/nm1096-1096 (1996).

26 Koshiba, T. et al. Expression of Stromal Cell-derived Factor 1 and CXCR4 Ligand Receptor System in Pancreatic Cancer: A Possible Role for Tumor Progression. Clin. Cancer Res. 6, 3530-3535 (2000).

27 Wang, L. et al. Donor bone-marrow CXCR4+ Foxp3+ T-regulatory cells are essential for costimulation blockade-induced long-term survival of murine limb transplants. Sci. Rep. 10, 9292, doi:10.1038/s41598-020-66139-x (2020).

28 Gobert, M. et al. Regulatory T cells recruited through CCL22/CCR4 are selectively activated in lymphoid infiltrates surrounding primary breast tumors and lead to an adverse clinical outcome. Cancer Res. 69, 2000-2009, doi:10.1158/0008-5472.Can-08-2360 (2009).

29 Wu, A. et al. Combination anti-CXCR4 and anti-PD-1 immunotherapy provides survival benefit in glioblastoma through immune cell modulation of tumor microenvironment. J. Neurooncol. 143, 241-249, doi:10.1007/s11060-019-03172-5 (2019).

\section{Tables}

Table 1. Clinicopathologic findings of 31 cases of conjunctival squamous cell Carcinoma. 


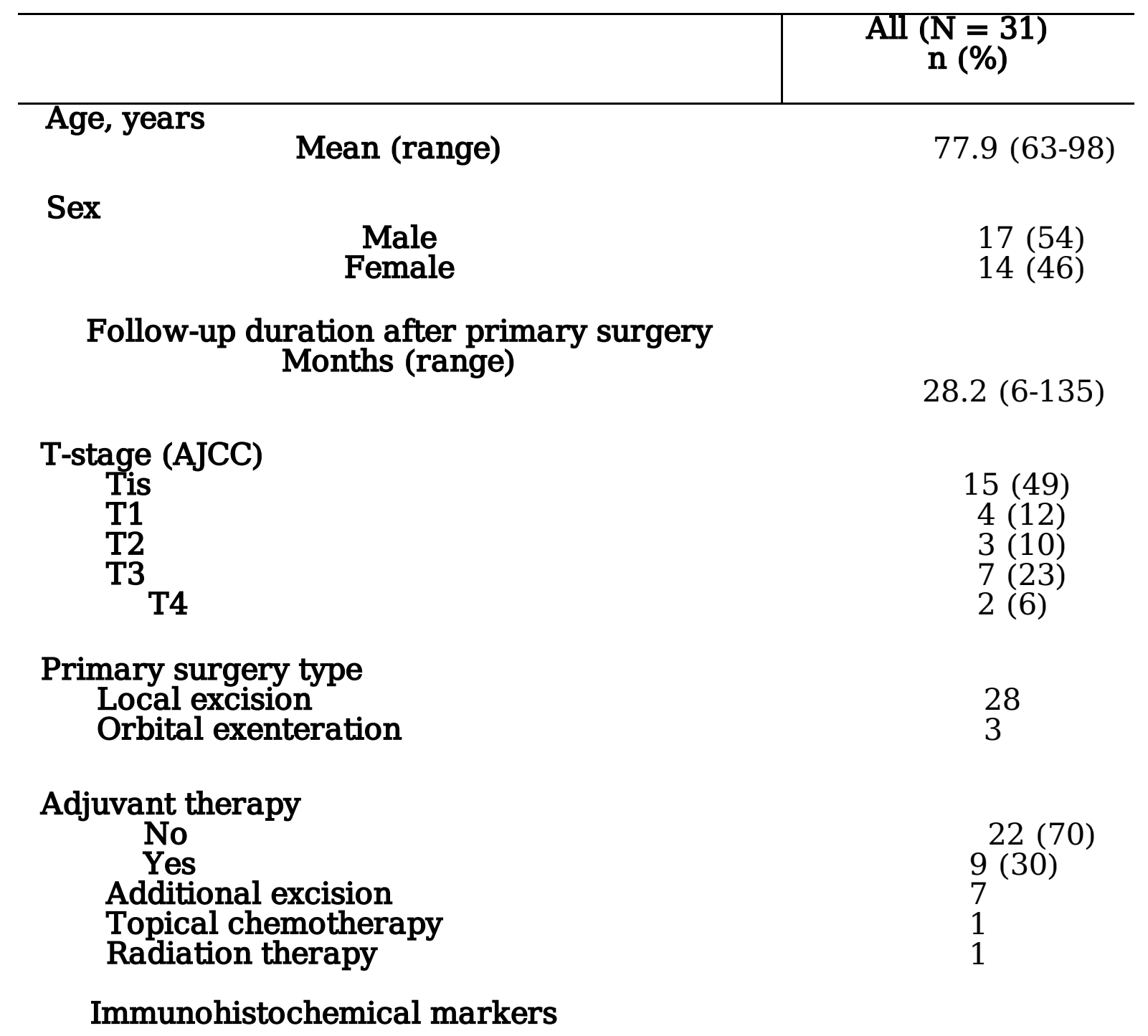

Ki67 labeling index

$\geq 50 \%$

$<50 \%$

Outcome

Orbital exenteration

Yes

No

Local recurrence after curative therapy

Yes

No

Metastasis

Distant

Regional + Distant

Regional

None

$7(22)$

24(78)

$5(16)$

$26(84)$

$7(22)$
$24(78)$

$0(0)$

$2(6)$

1 (3)

$27(91)$

$3(10)$

Vital status at last follow-up

Deceased 


\section{Cause of death}

Conjunctival SCC (metastasis)

Table 2. FOXP3 and CD8 staining patterns.

\begin{tabular}{|l|l|l|l|l|l|l|}
\hline & $\begin{array}{l}\text { FOXP3 } \\
\text { surface }\end{array}$ & $\begin{array}{l}\text { FOXP3 } \\
\text { tumor } \\
\text { central }\end{array}$ & $\begin{array}{l}\text { FOXP3 } \\
\text { around } \\
\text { vessels }\end{array}$ & $\begin{array}{l}\text { CD8 } \\
\text { surface }\end{array}$ & $\begin{array}{l}\text { CD8 } \\
\text { tumor } \\
\text { central }\end{array}$ & $\begin{array}{l}\text { CD8 } \\
\text { around } \\
\text { vessels }\end{array}$ \\
\hline $\begin{array}{l}\text { Tis } \\
\mathbf{n}=\end{array}$ & $0.20 \pm 0.32$ & $0.46 \pm 0.56$ & $1.0 \pm 0.93$ & $0.60 \pm 0.72$ & $0.86 \pm 0.69$ & $2.0 \pm 0.40$ \\
\hline $\begin{array}{l}\text { Tadv } \\
\mathbf{n}=\end{array}$ & $0.86 \pm 0.92$ & $2.13 \pm 2.07$ & $2.60 \pm 2.57$ & $0.20 \pm 0.21$ & $0.60 \pm 0.64$ & $1.26 \pm 1.28$ \\
\hline 16 & & & & & & \\
\hline$P$ & $<0.01 *$ & $<0.01 *$ & $<0.01 *$ & 0.12 & 0.35 & $<0.01 *$ \\
\hline
\end{tabular}

Tis, carcinoma in situ; Tadv, advanced carcinoma.

$*$ Un-paired $t$-test.

Table 3. FOXP3/CD8 ratio. 


\begin{tabular}{|l|c|c|c|}
\hline & $\begin{array}{l}\text { FOXP3/CD8 } \\
\text { ratio (SF) }\end{array}$ & $\begin{array}{l}\text { FOXP3/CD8 } \\
\text { ratio (TC) }\end{array}$ & $\begin{array}{l}\text { FOXP3/CD8 } \\
\text { ratio (AV) }\end{array}$ \\
\hline $\begin{array}{l}\text { Tis } \\
\mathbf{n = 1 5}\end{array}$ & $0.37 \pm 0.50$ & $0.33 \pm 0.48$ & $0.57 \pm 0.67$ \\
\hline $\begin{array}{l}\text { Tadv } \\
\mathbf{n}=16\end{array}$ & $0.20 \pm 0.21$ & $0.65 \pm 0.74$ & $2.25 \pm 2.28$ \\
\hline $\boldsymbol{P}$ & 0.36 & 0.31 & $<0.01^{*}$ \\
\hline
\end{tabular}

AV, around vessels; SF, surface; TC, tumor central; Tis, carcinoma in situ; Tadv, advanced carcinoma.

$*$ Un-paired $t$-test.

Table 4. Gene expression-associated cancer progression between Tis and Tadv. 


\begin{tabular}{|c|c|c|c|c|c|c|}
\hline & $\begin{array}{c}\text { Log2 } \\
\text { fold } \\
\text { change }\end{array}$ & $\begin{array}{l}\text { Std } \\
\text { error } \\
(\log 2)\end{array}$ & $\begin{array}{c}\text { Lower } \\
\text { confidence } \\
\text { limit }(\log 2)\end{array}$ & $\begin{array}{c}\text { Upper } \\
\text { confidence } \\
\text { limit (log2) }\end{array}$ & $P$-value & probe.ID \\
\hline $\begin{array}{l}\text { CXCR4- } \\
\text { mRNA }\end{array}$ & $\underline{3.93}$ & 0.668 & 2.62 & 5.24 & $<0.01 *$ & NM_003467.2:1335 \\
\hline $\begin{array}{l}\text { EGFR- } \\
\text { mRNA }\end{array}$ & -0.51 & 0.32 & -1.14 & 0.116 & 0.162 & NM_201282.1:360 \\
\hline $\begin{array}{l}\text { HIF1A- } \\
\text { mRNA }\end{array}$ & $\underline{1.62}$ & 0.413 & 0.813 & 2.43 & $<0.01 *$ & NM_001530.2:1985 \\
\hline $\begin{array}{l}\text { ICAM1- } \\
\text { mRNA }\end{array}$ & 1.11 & 0.693 & -0.248 & 2.47 & 0.16 & NM_000201.2:2253 \\
\hline $\begin{array}{l}\text { IL6- } \\
\text { mRNA }\end{array}$ & $\underline{4.03}$ & 1.1 & 1.88 & 6.19 & $0.0145^{*}$ & NM_000600.1:220 \\
\hline $\begin{array}{l}\text { MAPK1- } \\
\text { mRNA }\end{array}$ & -0.84 & 0.472 & -1.76 & 0.0856 & 0.126 & NM_138957.2:430 \\
\hline $\begin{array}{l}\text { MMP2- } \\
\text { mRNA }\end{array}$ & 1.89 & 0.939 & 0.044 & 3.73 & 0.0916 & NM_004530.2:2360 \\
\hline $\begin{array}{l}\text { MMP9- } \\
\text { mRNA }\end{array}$ & 2.85 & 1.17 & 0.549 & 5.15 & 0.0513 & NM_004994.2:1530 \\
\hline $\begin{array}{l}\text { NFKB1- } \\
\text { mRNA }\end{array}$ & $-\underline{2.32}$ & 0.344 & -3 & -1.65 & $<0.01 *$ & NM_003998.2:1675 \\
\hline $\begin{array}{l}\text { NOTCH1- } \\
\text { mRNA }\end{array}$ & 0.219 & 0.493 & -0.746 & 1.18 & 0.672 & NM_017617.3:735 \\
\hline $\begin{array}{l}\text { ROCK1- } \\
\text { mRNA }\end{array}$ & $\underline{0.731}$ & 0.273 & 0.196 & 1.27 & $0.0367 *$ & NM_005406.1:2660 \\
\hline $\begin{array}{l}\text { STAT3- } \\
\text { mRNA }\end{array}$ & 0.834 & 0.385 & 0.0799 & 1.59 & 0.0733 & NM_139276.2:4535 \\
\hline $\begin{array}{l}\text { ROCK2- } \\
\text { mRNA }\end{array}$ & 0.503 & 0.264 & -0.0152 & 1.02 & 0.106 & NM_004850.3:3140 \\
\hline & & & & & & \\
\hline
\end{tabular}




\begin{tabular}{|l|c|c|c|c|c|c|}
$\begin{array}{l}\text { TGFBI- } \\
\text { mRNA }\end{array}$ & -0.495 & 0.977 & -2.41 & 1.42 & 0.631 & NM_000358.2:2030 \\
\hline $\begin{array}{l}\text { TNFSF10- } \\
\text { mRNA }\end{array}$ & 0.00102 & 0.724 & -1.42 & 1.42 & 0.999 & NM_003810.2:115 \\
\hline $\begin{array}{l}\text { VEGFA- } \\
\text { mRNA }\end{array}$ & -0.375 & 0.731 & -1.81 & 1.06 & 0.626 & NM_001025366.1:1325 \\
\hline $\begin{array}{l}\text { WNT5A- } \\
\text { mRNA }\end{array}$ & -1.68 & 0.697 & -3.04 & -0.31 & 0.0529 & NM_003392.3:475 \\
\hline
\end{tabular}

Tis, carcinoma in situ $(n=4)$; Tadv, advanced carcinoma $(n=4)$.

$*$ Un-paired $t$-test $(P<0.05)$. Statistically significant differences are underlined.

Table 5. Relationship between progression-free survival and various clinicopathologic and molecular factors.

\begin{tabular}{|c|c|c|c|c|}
\hline \multicolumn{5}{|c|}{ Univariate analysis } \\
\hline Variable & $\mathbf{N}=31$ & HR & $95 \% \mathrm{CI}$ & $P$ \\
\hline Age & Mean 77.9 years & 1.305 & 0.904-1.882 & 0.155 \\
\hline Sex & Male 17, Female 14 & 3.294 & $0.339-32.035$ & 0.304 \\
\hline T-stage (AJCC) & Tis: $15,>\mathrm{T} 1: 16$ & 3.428 & $0.356-33.031$ & 0.287 \\
\hline Ki67 index (\%) & Average: 30.58\% & 1.017 & 0.987-1.049 & 0.266 \\
\hline FOXP3/CD8 ratio & Tis: $0.57 \mathrm{~T}>1: 2.25$ & $\underline{2.684}$ & $1.005-7.170$ & 0.049* \\
\hline
\end{tabular}

AJCC, American Joint Committee on Cancer; CI, confidence interval; HR, hazard ratio.

Statistically significant association is underlined.

*Cox proportional hazard model.

\section{Figures}



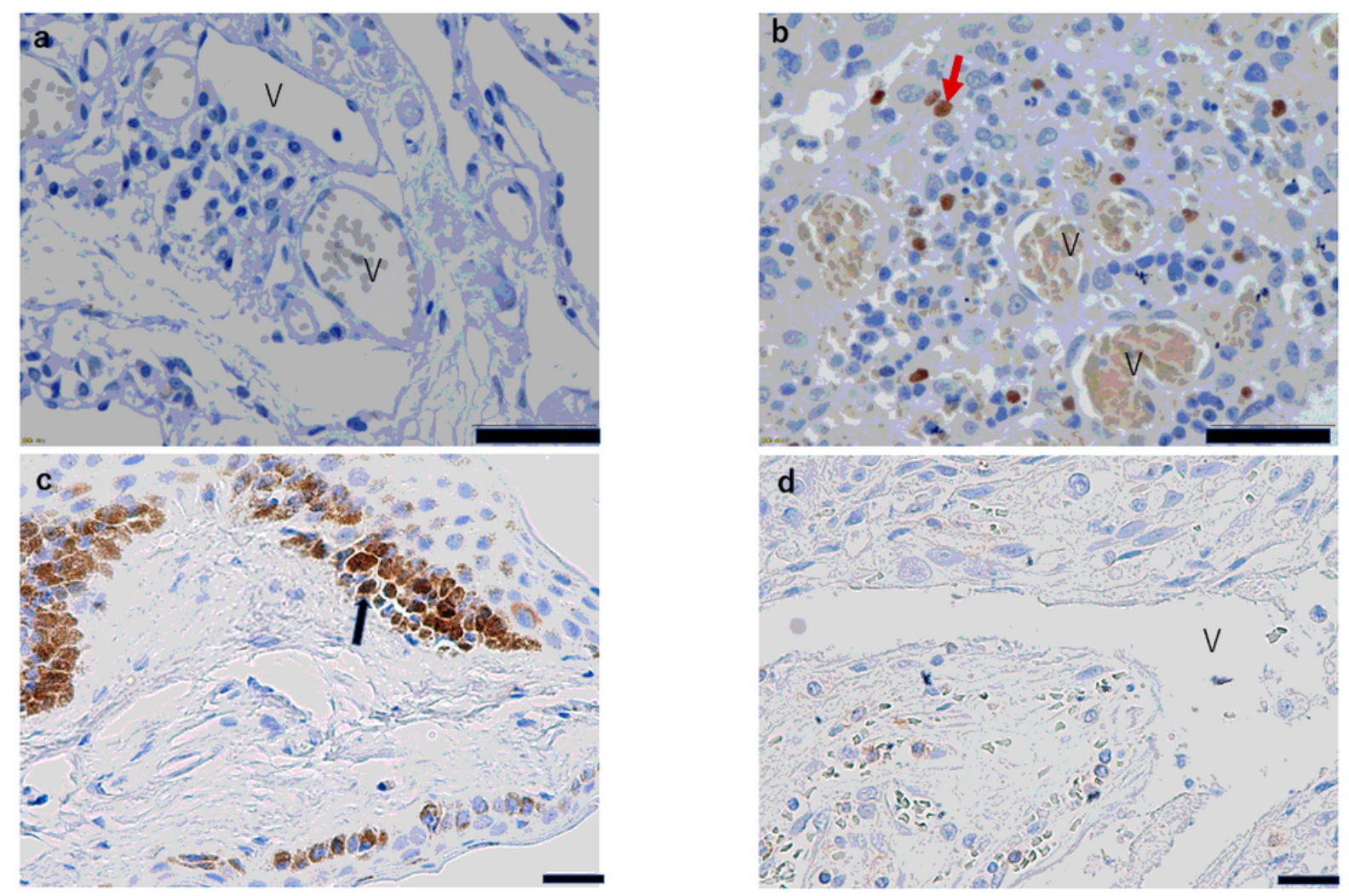

\section{Figure 1}

FOXP3 and CD8 expression in conjunctival SCC tumor tissue. Weak FOXP3 staining (score: 1) (a) and strong FOXP3 staining (b) (scale bar: $50 \mu \mathrm{m}$ ). Very strong CD8 staining (score: 3 ) (c) and weak CD8 staining (score: 1) (d) (scale bar: $20 \mu \mathrm{m}$ ). V: vascular vessels. Red arrow in (b) denotes FOXP3-positive lymphocyte. Black arrow in (c) denotes CD8-positive lymphocyte 


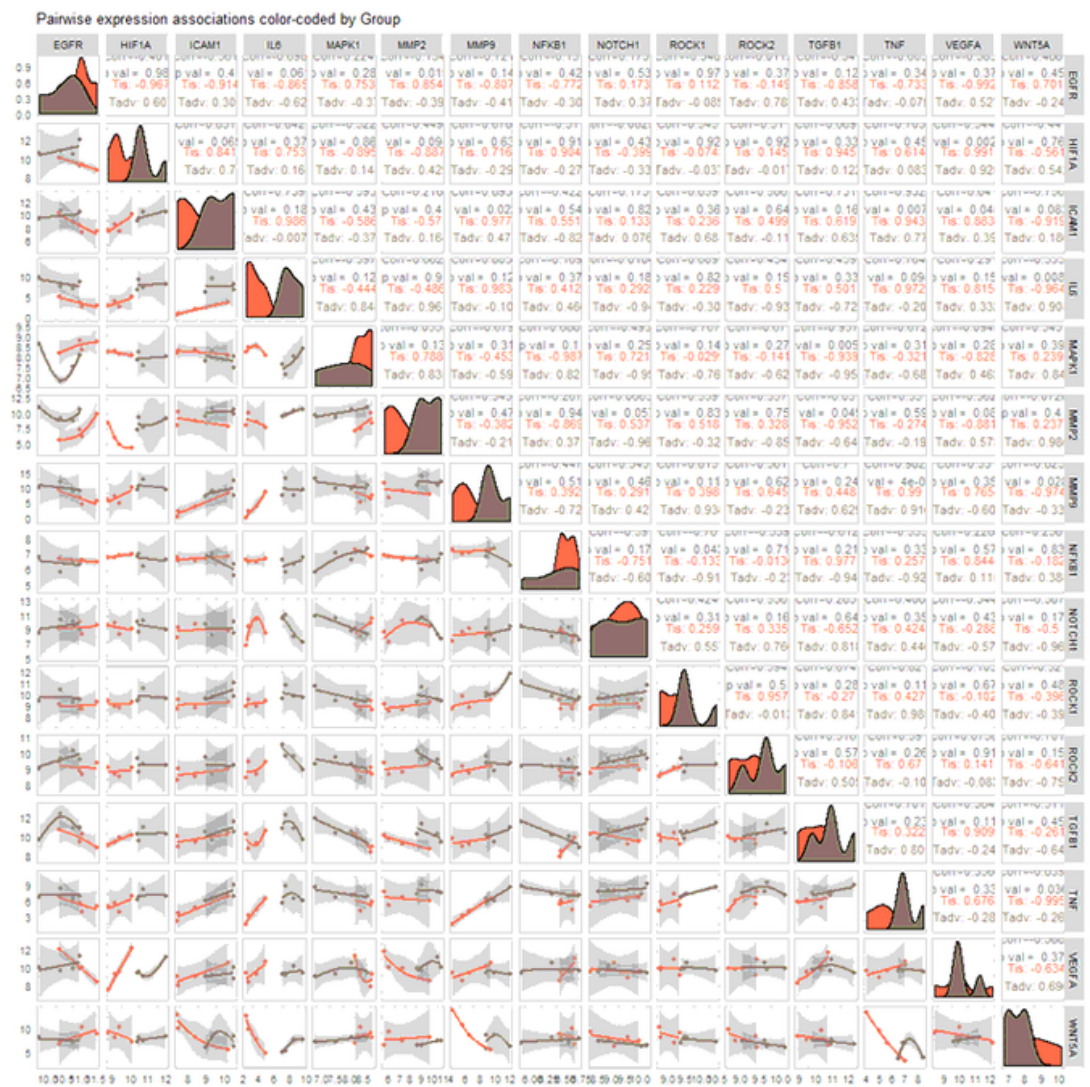

Figure 2

Correlation plots of pairwise expression associations, color-coded by group between the in situ (Tis) and invasive carcinoma (Tadv) groups according to AJCC T-scale grading ( $\mathrm{n}=4$ in each group).
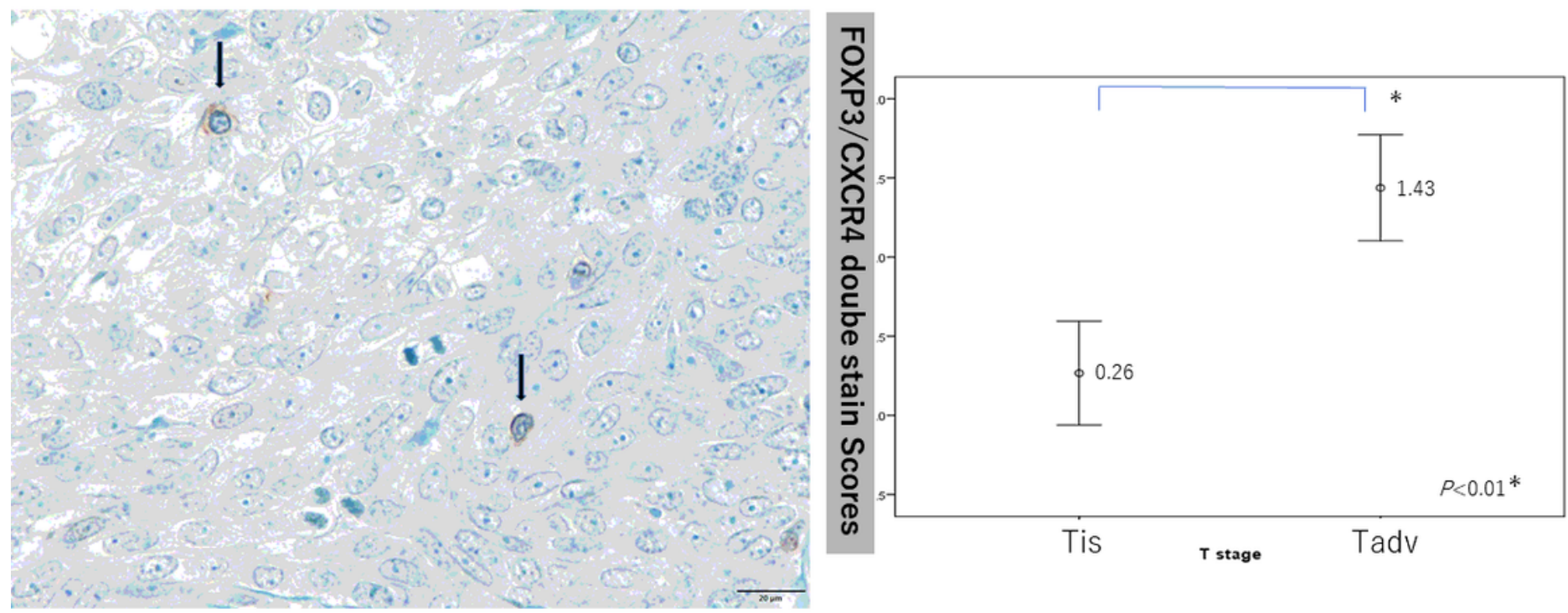


\section{Figure 3}

Double CXCR4/FOXP3 immunostaining to evaluate CXCR4-positive Treg infiltration in whole tissue samples. (Left) Black arrow: double staining, nuclear (blue: FOXP3), and cytoplasm and cell membrane (brown: CXCR4) (scale bar: $20 \mu \mathrm{m}$ ). (Right) A higher proportion of Tadv cancers expressed CXCR (score: 1.43) compared with Tis cancers (score: 0.26$)(P<0.01)$

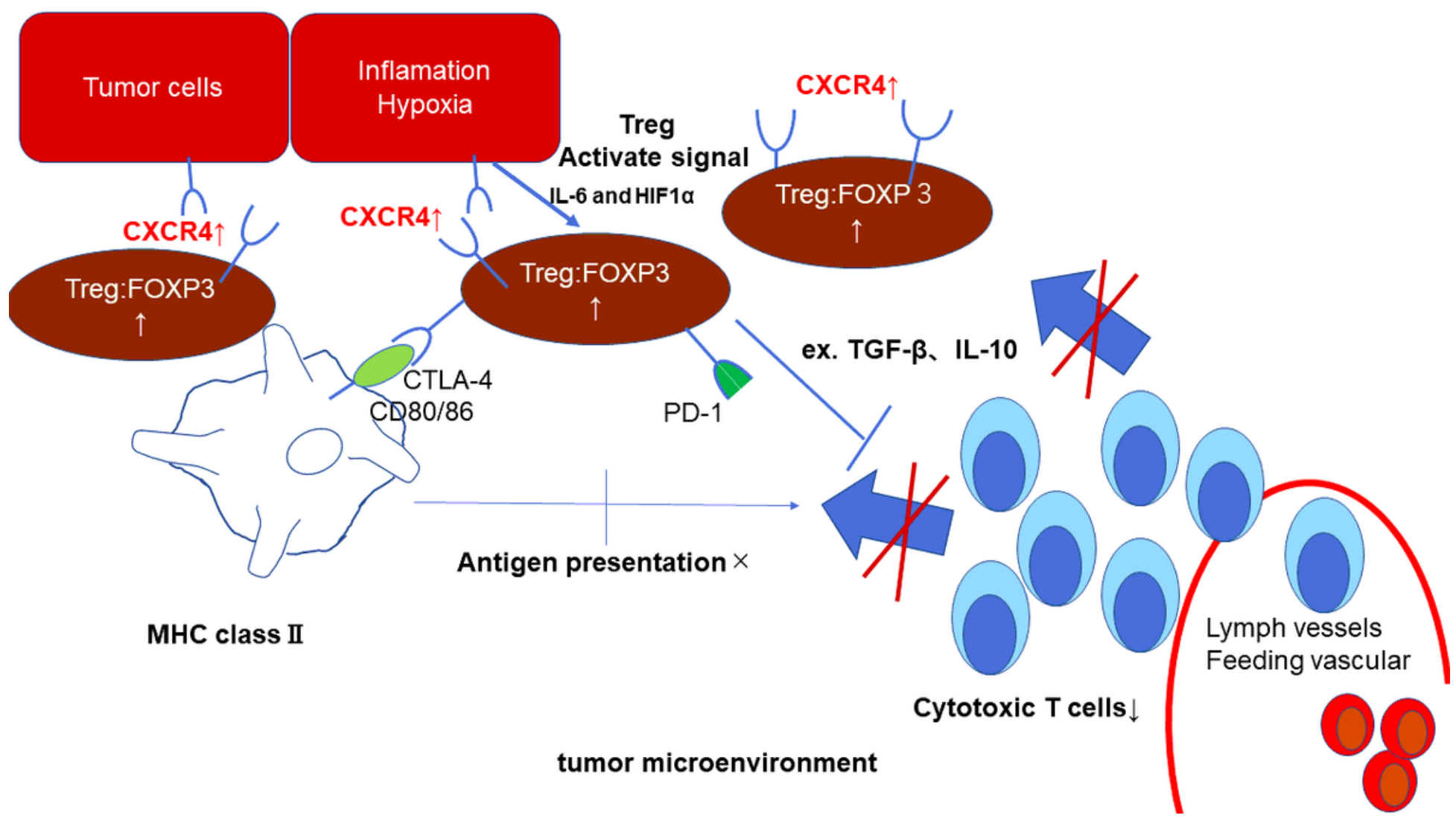

Figure 4

Schematic illustration of hypothetical tumor immunity process in conjunctival squamous cell carcinoma 